\title{
Commentary Hippocrates is alive and weaning in Brazil
} Nalan Adıgüzel11,2, Gökay Güngör ${ }^{1,2}$ and Martin J Tobin ${ }^{1}$

\begin{abstract}
1'Division of Pulmonary and Critical Care Medicine, Edward Hines Jr Veterans Affairs Hospital and Loyola University of Chicago Stritch School of Medicine, 5th Avenue and Roosevelt Road, Hines, IL 60141, USA

${ }^{2}$ Respiratory Intensive Care Unit, Süreyyapașa Chest Diseases and Chest Surgery Teaching and Research Hospital, Basibüyük Street, Istanbul, 34844 Turkey
\end{abstract}

Corresponding author: Martin J Tobin, mtobin2@lumc.edu

Published: 18 May 2009

Critical Care 2009, 13:142 (doi:10.1186/cc7746)

This article is online at http://ccforum.com/content/13/3/142

(c) 2009 BioMed Central Ltd

See related research by Taniguchi et al., http://ccforum.com/content/13/1/R6

\begin{abstract}
In a group of postoperative patients, Taniguchi and coworkers compared the effect of a computerized system for weaning against 'manual care'. The computerized system involved automatic adjustments to the level of pressure support to achieve a target respiratory rate. Manual care involved adjustments to the level of pressure support to keep the ratio of respiratory frequency to tidal volume below 80 . The duration of ventilator weaning was equivalent with the two approaches. The level of pressure support, however, was lower with manual care than with computerized ventilation. The study adds support to the notion that ventilator duration is shortened when weaning is contemplated the earliest possible time. The findings also emphasize the importance of the Hippocratic dictum that patient outcome is improved when care is individualized rather than delivered according to a protocol.
\end{abstract}

In a previous issue of Critical Care, Taniguchi and coworkers [1], from São Paulo, Brazil, report a new study on ventilator weaning. The study represents a part of the evolution of the approach to weaning that has occurred over the past several decades [2]. Thirty years ago patients were weaned without any guiding framework; few of the mechanisms that lead to weaning failure (or success) had been discovered. Subsequently, attention focused on the use of tests to predict weaning outcome. Later still, specific techniques for performing a weaning trial were compared. In recent years, attention has turned to the use of protocols in weaning, although one could argue that this step represents regression rather than an advance [3].

In their study, Taniguchi and coworkers [1] compared the effect of a computerized system for weaning against 'manual care'. The computerized system is called 'mandatory rate ventilation', and is available on the Taema-Horus ventilator (Taema, Antony, France). The algorithm automatically adjusts the level of pressure support to achieve a target respiratory rate. If the patient's respiratory rate (averaged over four respiratory cycles) exceeds the target by more than three breaths per minute, then the level of pressure support is increased by $1 \mathrm{~cm} \mathrm{H}_{2} \mathrm{O}$, and vice versa. Manual care involved adjustments to the level of pressure support to keep the ratio of respiratory frequency to tidal volume below 80 .

The Brazilian researchers found no difference in the duration of weaning between the two approaches. The level of pressure support was lower with manual care than with computerized ventilation (over at least the first 3 hours of the study). They also reported more complications with the computerized system, although this is predicated by a peculiar manner of defining complications.

The results contrast with those reported by Lellouche and coworkers [4], who reported significant decreases in the duration of weaning, total ventilator duration, and intensive care stay with their computerized system as compared with usual care, which involved the use of paper-based protocols. A number of factors may account for the difference in outcomes between the two studies. The computer algorithm in the ventilator used by Lellouche and coworkers may be superior to that used by the Brazil researchers. The patient populations also differed, because Taniguchi and coworkers [1] confined their study to postoperative patients. Furthermore, the Brazilian clinical team knew that they were competing against a machine and, importantly, against a machine that they did not help to create. Like the outcome reported by Taniguchi and coworkers [1], a group of Australian researchers found that computerized weaning was not superior to usual care [5], despite using the same system as that employed by Lellouche and coworkers.

$f / V_{T}=$ ratio of respiratory frequency to tidal volume. 
When conducting scientific experiments, including randomized trials, the control arm is organized to serve as bland background, so that the intervention arm can shine more brightly. The control arm is viewed as the 'boring' part of the trial. In critical care medicine, we have learned that the control arm is often more interesting than the intervention arm. The report from Brazil [1] is another example of this pattern. In the control group, the medical team manually adjusted the level of pressure support every 30 minutes to keep the ratio of respiratory frequency to tidal volume $\left(f / V_{T}\right)$ below 80 [6]. The data presented in Figure 3 of their report are striking; the level of pressure support set by the medical team was impressively lower $(P<0.001)$ than the level selected by the computerized algorithm.

The development of ventilators that enable computerized weaning draw attention to a major problem in ventilator management - clinicians are slow to initiate weaning [2]. In studies of $f / V_{T}$ as a weaning predictor, the pre-test probability of weaning success exceeded $75 \%$ in more than half of the studies [7]. For optimal results with a screening test such as $\mathrm{f} / \mathrm{V}_{\mathrm{T}}$, it must be conducted at a time when a negative result is far more likely than a positive one - when the pre-test probability of weaning success will be much less than $50 \%$. The algorithms in the computerized weaning systems do this automatically. We suspect that the clinicians in the control arms of the Brazilian [1] and Australian studies [5] were aware of the results of the study reported by Lellouche and coworkers [4], and tacitly incorporated the broader message of that study into their clinical practice. The key message from the work of Lellouche and coworkers is that the duration of ventilator support is shorter if weaning is contemplated at the earliest possible time during the period of mechanical ventilation, and repeated assessments of patients expedite the process.

Difficulties in ventilator weaning can result from respiratory muscle weakness, abnormal respiratory mechanics, impaired gas exchange, cardiac dysfunction, psychological distress and other factors [3]. Determining the reason for difficulty in weaning in a particular patient requires an astute clinician. Few aspects of critical care medicine are more dependent on diagnostic acumen and individualized care [2]. Hippocrates is best remembered for his admonition against harm (primum non nocere), but he equally stressed the importance of individualized care [8]. Individualized care is the antithesis of protocolized care, which was promulgated by the EvidenceBased Medicine Task Force on weaning [9]. Numerous randomized trials have now shown that protocols hinder rather than expedite weaning. The new data from Brazil re-emphasize that a 2,000-year-old message still resonates - patients do better when clinicians 'handle' them as individuals.

\section{Competing interests}

MJT receives royalties for "Principles and Practice of Mechanical Ventilation", published by McGraw Hill. He does not receive financial support from ventilator or medical device companies. NA and GG have no competing interests.

\section{Reference}

1. Taniguchi C, Eid RC, Saghabi C, Souza R, Silva E, Knobel E, Paes T, Barbas CS: Automatic versus manual pressure support reduction in the weaning of post-operative patients: a randomized controlled trial. Crit Care 2009, 13:R6.

2. Tobin MJ: Remembrance of weaning past: the seminal papers. Intensive Care Med 2006, 32:1485-1493.

3. Tobin MJ, Jubran A: Weaning from mechanical ventilation. In Principles and Practice of Mechanical Ventilation, 2nd ed. Edited by Tobin MJ. New York, NY: McGraw-Hill, Inc.; 2006:1185-1220.

4. Lellouche F, Mancebo J, Jolliet P, Roeseler J, Schortgen F, Dojat M, Cabello B, Bouadma L, Rodriguez P, Maggiore S, Reynaert M, Mersmann S, Brochard L: A multicenter randomized trial of computer-driven protocolized weaning from mechanical ventilation. Am J Respir Crit Care Med 2006, 174:894-900.

5. Rose L, Presneill JJ, Johnston L, Cade JF: A randomised, controlled trial of conventional versus automated weaning from mechanical ventilation using SmartCare/PS. Intensive Care Med 2008, 34:1788-1795.

6. Yang K, Tobin MJ: A prospective study of indexes predicting the outcome of trials of weaning from mechanical ventilation. N Engl J Med 1991, 324:1445-1450.

7. Tobin MJ, Jubran A: Variable performance of weaning-predictor tests: role of Bayes' theorem and spectrum and test-referral bias. Intensive Care Med 2006, 32:2002-2012.

8. Nuland SB: The totem of medicine: Hippocrates. In Doctors: the biography of medicine. New York: Alfred A Knopf; 1988:3-30.

9. Ely EW, Meade MO, Haponik EF, Kollef MH, Cook DJ, Guyatt GH, Stoller JK: Mechanical ventilator weaning protocols driven by nonphysician health-care professionals: evidence-based clinical practice guidelines. Chest 2001, 120:454S-463S. 